\title{
Difference in prevalence of exertional oscillatory ventilation between healthy subjects and patients with cardiovascular disease
}

\author{
Ugo Corrà $^{1}$, Massimo Piepoli², Andrea Giordano ${ }^{3}$ \\ ${ }^{1}$ Division of Cardiology, ICS Maugeri, Institute of Veruno; ${ }^{2}$ Heart Failure Unit, Cardiology Department, Polichirurgico \\ Hospital G. Da Saliceto, Piacenza; ${ }^{3}$ Bio-engineering Service, ICS Maugeri, Institute of Veruno, Italy
}

\begin{abstract}
Exercise oscillatory ventilation (EOV) is an ominous sign in heart failure due to reduced left ventricular ejection fraction
\end{abstract}

\footnotetext{
Correspondence: Dr. Ugo Corrà, Head of the Exercise Pathophysiology Laboratory, ICS Maugeri, Veruno Institute, Via per Revislate 13, 28010, Veruno (NO), Italy.

Tel. +39.0322.884711- Fax: +39.0322.830294.

E-mail: ugo.corra@icsmaugeri.it
}

Key words: Cardiopulmonary exercise testing; exertional oscillatory ventilation; heart failure.

Contributions: All the authors made a substantive intellectual contribution. All the authors have read and approved the final version of the manuscript and agreed to be accountable for all aspects of the work.

Acknowledgments: The authors are grateful to Rosemary Allpreess for her careful revision of the English manuscript.

Study association: This study is not associated with any post-graduation program.

Conflict of interest: The authors declare that they have no competing interests, and all authors confirm accuracy.

Funding: There were no external funding sources for this study.

Availability of data and materials: The datasets used and/or analyzed during the current study are available from the corresponding author on reasonable request.

Ethics approval and consent to participate: The study was carried out in accordance with the Code of Ethics of the World Medical Association (Declaration of Helsinki).

Informed consent: An informed written consent was obtained from all participants, and the protocol was approved by the ICS Maugeri Central Ethics Committee.

Received for publication: 18 December 2019.

Accepted for publication: 1 March 2020.

${ }^{\circ}$ Copyright: the Author(s), 2020

Licensee PAGEPress, Italy

Monaldi Archives for Chest Disease 2020; 90:1203

doi: 10.4081/monaldi.2020.1203

This article is distributed under the terms of the Creative Commons Attribution Noncommercial License (by-nc 4.0) which permits any noncommercial use, distribution, and reproduction in any medium, provided the original author(s) and source are credited.
(HFrEF) whatever it is represented. But EOV is detected also in normal healthy individuals and in other cardiovascular disease (CVD) patients, however, its prevalence in these is not completed clear. The aim was to describe the occurrence of EOV in healthy subjects and the overall population all CVD patients who performing symptomlimited cardiopulmonary exercise testing (CPET). Healthy subjects were divided in athletes and normal subjects, while, CVD patients were subdivided into: i) t hose with preserved left ventricular ejection fraction (LVEF); ii) those with mild to moderate impairment of LVEF (41-49\%); iii) those with severe impairment of LVEF ( $\leq 40 \%$ ); iv) HFrEF or with preserved LVEF (HFpEF); and iv) patients after heart transplantation (HXT). EOV was observed only in CVD patients and in those with depressed LVEF; the prevalence of EOV was observed $1.9 \%(3 / 55)$ those with mild to moderate impairment of LVEF (41-49\%), 3.4\% (56/1613) those with severe impairment of LVEF ( $\leq 40 \%)$, and $7.3 \%(214 / 2903)$ in HFrEF); no EOV was observed in CVD with preserved LVEF. Kremser's EOV was observed in patients, and, particularly, in those with systolic function impairment. Moreover, as EOV impacts prognosis in $\mathrm{HFrEF}$, its occurrence can modify prognostic-decision models. Even though, EOV prevalence was derived from largest single center population, more studies are needed to tackle the EOV prevalence in different CVD conditions and in normal subjects.

\section{Introduction}

Exercise oscillatory ventilation (EOV) is an atypical exertional respiratory response [1], characterized waxing and waning in ventilation (VE). EOV is frequently detected in heart failure due to reduced left ventricular ejection fraction (HFrEF) [2,3], and it is an ominous sign, whatever it is represented [3]. EOV is discovered also in normal healthy individuals [4] and in other cardiovascular disease (CVD) patients [5]. Though, EOV prevalence is not clear, yet.

The principal aims of this study were 1) to describe the occurrence of EOV in the overall population, healthy subjects and CVD patients, with or without history of heart failure (HF), performing symptom-limited cardiopulmonary exercise testing (CPET) 2) to assess of EOV prevalence in $\mathrm{HFrEF}$, and resting factors that might predict this abnormal respiratory phenomenon.

\section{Patients and Methods}

\section{Data source}

This was a retrospective study of data collected prospectively for prognostic assessment purposes; here, follows data on EOV 
prevalence. The study was based on patient medical records from the ergo-spirometry laboratory of the Istituti Clinici Scientifici Maugeri, IRCCS Scientific Institute of Veruno (NO), Italy; CPET data were recruited from September 15, 1995 to December 31, 2016 (12 years). All records originated from the ergo-spirometry laboratory of Veruno, and, subjects' and patients' documents and statistics were, anonymously, used. A note was kept about demographic, clinical, echocardiographic parameters, as well as about pharmacological treatment.

The study was carried out in accordance with the Code of Ethics of the World Medical Association (Declaration of Helsinki). An informed written consent was obtained from all participants, and the protocol was approved by the ICS Maugeri Central Ethics Committee.

\section{Study population}

Healthy subjects performed symptom-limited CPET for functional assessment, while, for CVD patients, CPET was executed for both functional and prognostic estimations. Those with HF were categorized, in order to the history or recent $(<3$ months) existence of signs or symptoms of HF, as described ESC guidelines [6]. CVD patients were subdivided, according left ventricular ejection fraction (LVEF) and symptoms/signs of HF. They were classified as follows: i) those with preserved left ventricular ejection fraction $(\mathrm{LVEF}=\geq 50 \%$ ), those with mild to moderate impairment of LVEF (41-49\%), and those with severe impairment of LVEF $(\leq 40 \%)$; all above-mentioned patients had no HF symptoms or signs, ii) HF patients (these patients were characterized to have had symptoms or signs of HF, according ESC guideline) with reduced LVEF (HFrEF) or with preserved LVEF (HFpEF), iii) patients after heart transplantation (HXT). HXT patients were evaluated one month after surgery.

Exclusion criteria were: 1) myocardial infarction, myocardial revascularization or unstable angina, one month before the study; 2) ventricular arrhythmias induced by exercise (sustained ventricular tachycardia or fibrillation); 3) severe aortic valvular stenosis; 4) concomitant diseases that might limit the exercise capacity assessment (either in healthy subjects or CVD patients); 5) cardiac strategies scheduled; 6) unwillingness to provide informed consent.

Eligibility criteria were: 1 ) the ability to perform a CPET, limited by fatigue or dyspnea, with a peak respiratory exchange ratio (RER) $\geq 1.00$ [7], and 2) for CVD/HFrEF patients, clinical/pharmacological stability 1 month before CPET.

\section{Cardiopulmonary exercise testing}

CPET was performed on a bicycle ergometer with a ramp protocol for all, healthy subjects and CVD patients. For health subjects, a 20 Watts, every one-minute ramp protocol was managed, while for CVD patients a 10 Watts every one minute was arranged. The CPET was conducted with breath-by-breath respiratory gas exchange (Sensormedics, Vmax 29, Yorba Linda, CA, USA).

Peak $\mathrm{VO}_{2}$ was recorded as the mean value of $\mathrm{VO}_{2}$ during the last 60 seconds of the test, while the relationship between ventilation (VE) and carbon dioxide $\left(\mathrm{VCO}_{2}\right)$ was computed as a linear regression function from the whole exercise period $\left(\mathrm{VE} / \mathrm{VCO}_{2}\right.$ slope) [8], and percentage of predicted peak $\mathrm{VO}_{2}\left(\mathrm{VO}_{2} \%\right)$ was processed, according to Wasserman et al.'s formula [9]. In the post-test phase,
VE (L/min.) was displayed on an expanded time scale, and EOV was launched, applying Kremser et al.'s criteria [10]. We traditionally adopted Kremser's method (mainly for outcome reasons, in HFrEF patients); we persisted to use this procedure to harmonize CPET data. This method implies i) cyclic fluctuations in VE lasting longer than $66 \%$ of the whole exercise duration, and ii) amplitude of each single VE oscillation more than $15 \%$ of the average value at rest. A typical EOV pattern is shown in Figure 1.

Blood pressure was measured manually at rest, every $3 \mathrm{~min}$ during incremental exercise, and at peak of exercise, while electrocardiogram and heart rate were monitored at rest and throughout exercise with at 1-min intervals. All CPETs were conducted on medical therapy.

CPET results were interpreted and revised by experienced and qualified cardiologists.

\section{Echocardiographic evaluation}

Trans-thoracic echocardiograms were performed within 4-6 days of CPET, and LVEF and trans-mitral deceleration time (DecT.) were calculated as described [11].

\section{Statistical analysis}

Continuous data was expressed as means \pm standard deviation (SD). Student's $t$-test for non-paired values was used to compare the means of groups for quantitative variables. For qualitative variables, the $\chi^{2}$ test with Yates' correction or Fisher's exact test, if necessary, was employed. Logistic regression univariate and multivariable analysis for EOV was performed in HFrEF; for multivariable inquiry, we used significant resting variable at univariate analysis. The level of statistical significance was set $\mathrm{p}$-value $<0.05$.

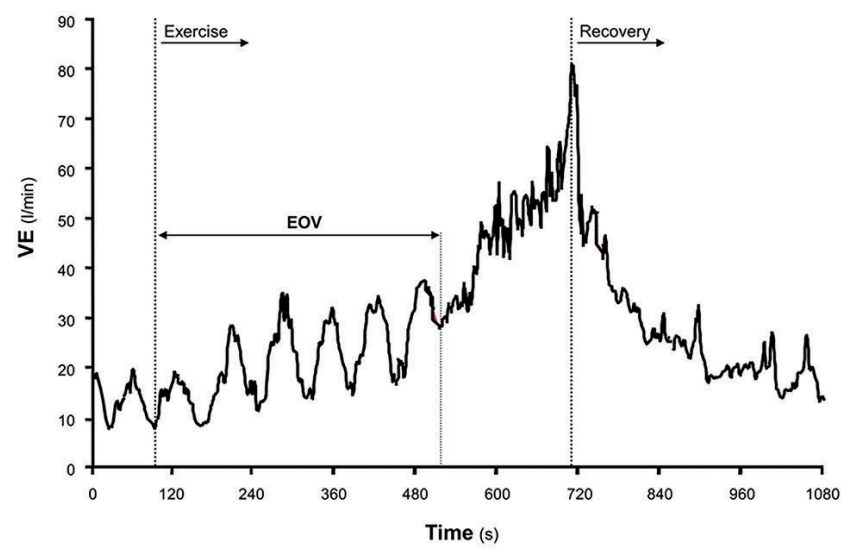

Figure 1. Representation of exertional oscillatory ventilation (EOV). Exercise represents when incremental exercise phase is organized (ramp fashion; 10 watts $/ 1$ min for patients; 20 Watts/ 1 min for healthy subjects). The exercise phase is anticipated by a warm-up pedaling period, usually lasting 1-2 min. The recovery phase is when incremental exercise is stopped. This phase lasts 6 min: it is composed by 1 -min pedaling phase at 10 watts, followed by a 5 -min resting phase, in setting position. VE, ventilation in $1 / \mathrm{min}$; Time, time of exercise phase in seconds. 
All calculations were performed using the STATA ${ }^{\circledR} 10$ system (StataCorp, College Station, TX, USA).

\section{Results}

We screened 5741 CPETs: 834 patients were excluded, because due to other limiting symptoms than other than fatigue or dyspnea ( $\mathrm{n}=197)$, peak RER lower $<1.00(\mathrm{n}=439)$, clinical/pharmacological stability not accomplished $(\mathrm{n}=198)$. The remaining
4907 CPETs formed the study population: 14 CVD patients had a LVEF $\geq 50 \%, 157$ CVD patients had LVEF between $41-49 \%$, and 1613 CVD patients showed a LVEF $\leq 40 \%$. HFrEF and HFpEF patients were 2903 and 55, respectively. Finally, HXT patients were 101 recipients (Table 1). Moreover, 64 healthy subjects (of these 35 were athletes) were screened. Overall, EOV was observed in 273 patients, $5.5 \%$ of total population: $1.9 \%$ in CVD patients with mild to moderate impairment, in $3.4 \%$ in those with severe left ventricular dysfunction and 7,3\% in HFrEF patients (Table 1). Therefore, EOV was witnessed only in CVD with LVEF impairment $(\mathrm{LVEF}<50 \%)$ or in $\mathrm{HFrEF}$ patients.

Table 1. Etiology and EOV occurrence in the overall study population.

\begin{tabular}{|c|c|c|}
\hline & Total number & EOV $(\%)$ \\
\hline Total CPETs analyzed and n (\%) of EOV & 4907 & $273(5.5)$ \\
\hline Athletes & 35 & $0(0)$ \\
\hline Other healthy subjects & 29 & $0(0)$ \\
\hline CVD with preserved LV systolic function (LVEF $\geq 50 \%$ ) & 14 & $0(0)$ \\
\hline HFpEF (LVEF $\geq 50 \%)$ & 55 & $0(0)$ \\
\hline CVD with mild to moderate impairment of LV systolic function (LVEF $>40$ and $<50 \%$ ) & 157 & $3(1.9)$ \\
\hline CVD with severe impairment of LV systolic function (LVEF $\leq 40 \%)$ & 1613 & $56(3.4)$ \\
\hline HFrEF (LVEF $\leq 40 \%)$ & 2903 & $214(7.3)$ \\
\hline Recent HXT & 101 & $0(0)$ \\
\hline
\end{tabular}

Values reported for continuous data are mean \pm SD, for raw data: number of patients (percentage of total). EOV, exertional oscillatory ventilation; CPET, cardiopulmonary exercise testing; CVD, cardiovascular disease; LVEF, left ventricular ejection fraction; HF, heart failure; HFpEF, heart failure with preserved LVEF; HFrEF, heart failure with reduced LVEF; HXT, heart transplantation.

Table 2. demographic, clinical, echocardiographic, CPET and medical characteristic of HFrEF patients with and without EOV.

\begin{tabular}{|c|c|c|c|}
\hline & With EOV ( $n=214)$ & Without EOV (n=2694) & p \\
\hline Age (years) & $61 \pm 9$ & $58 \pm 10$ & 0.0015 \\
\hline Male (\%) & $213(91)$ & $2347(80)$ & 0.0661 \\
\hline BMI $\left(\mathrm{kg} / \mathrm{m}^{2}\right)$ & $25 \pm 3$ & $26 \pm 4$ & 0.0016 \\
\hline $\begin{array}{l}\text { Etiology of HF } \\
\text { IHD (\%) } \\
\text { ICD (\%) } \\
\text { VHD (\%) }\end{array}$ & $\begin{array}{l}128(8) \\
83(7) \\
3(15)\end{array}$ & $\begin{array}{l}1510(92) \\
1162(93) \\
17(85)\end{array}$ & 0.2143 \\
\hline NYHA class & $2.0 \pm 0.6$ & $2.3 \pm 0.5$ & $<0.0001$ \\
\hline $\begin{array}{l}\text { NYHA class - Categories } \\
\text { NYHA } 1(\%) \\
\text { NYHA } 2(\%) \\
\text { NHYA } 3(\%) \\
\end{array}$ & $\begin{array}{l}10(2) \\
112(7) \\
92(13)\end{array}$ & $\begin{array}{c}463(98) \\
1667(93) \\
564(86)\end{array}$ & $<0.0001$ \\
\hline Sinus rhythm (\%) & $193(82)$ & $2475(84)$ & $<0.0001$ \\
\hline $\operatorname{LVEF}(\%)$ & $22 \pm 8$ & $27 \pm 8$ & $<0.0001$ \\
\hline DecT (msec) & $147 \pm 47$ & $171 \pm 54$ & $<0.0001$ \\
\hline Beta-blockers: n (\%) & $148(63)$ & $2013(75)$ & 0.0002 \\
\hline ACE-inhibitors: n (\%) & $214(92)$ & $2452(93)$ & 0.8140 \\
\hline Loop diuretics: $\mathrm{n}(\%)$ & $220(94)$ & $2401(89)$ & 0.0134 \\
\hline Loop diuretics daily dose & $50 \pm 12$ & $37 \pm 8$ & 0,2013 \\
\hline Peak $\mathrm{VO}_{2}(\mathrm{ml} / \mathrm{kg} / \mathrm{min})$ & $12.9 \pm 3$ & $15.3 \pm 4$ & $<0.0001$ \\
\hline Percentage of predicted peak $\mathrm{VO}_{2}(\%)$ & $48 \pm 14$ & $55 \pm 14$ & $<0.0001$ \\
\hline $\mathrm{VENCO}_{2}$ slope & $32 \pm 6$ & $38 \pm 10$ & $<0.0001$ \\
\hline Peak RER & $1.13 \pm 0.09$ & $1.14 \pm 0.09$ & 0.6061 \\
\hline
\end{tabular}

Mean value \pm SD for continuous values or percentage for categorial values. CPET, cardiopulmonary exercise testing; EOV, exertional oscillatory ventilation; BMI, body mass index; NYHA, New York Heart Association; $\mathrm{HF}$, heart failure; IHD, ischemic heart disease; ICD, idiopathic cardiomyopathy; VHD, valvular heart disease; LVEF, left ventricular ejection fraction; DecT, trans-mitral deceleration time; ACE, angiotensin converting enzyme; $\mathrm{VO}_{2}$, oxygen consumption; $\mathrm{VENCO}_{2}$ slope, exertional relationship between ventilation ( $\mathrm{VE}$ ) and carbon dioxide $\left(\mathrm{VCO}_{2}\right)$; $\mathrm{RER}$, respiratory exchange ratio (i.e., peak $\mathrm{VO} / 2 \mathrm{NCO} 2$ ratio). 
Table 3. Logistic regression analysis for EOV prediction in HFrEF, using significant resting variables.

\begin{tabular}{lcccc} 
& $\chi^{2}$ & P & OR & 95\% CI \\
LVEF & 24.45 & $<0.0001$ & 0.942 & $0.920-0.968$ \\
DecT & 5.19 & 0.0226 & 0.996 & $0.992-0999$ \\
\hline Age & 8.95 & 0.0028 & 1.027 & $1.009-1.044$ \\
BMI & 4.42 & 0.0355 & 0.965 & $0.925-0.997$ \\
\hline Sinus rhythm & 14.11 & 0.0002 & 0.490 & $0.266-0.660$ \\
NYHA class & 14.89 & $<0.0001$ & 1,729 & $1.309-2.848$ \\
\hline
\end{tabular}

OR, odds ratio; $\mathrm{CI}$, confidence of interval; see also Table 2 for abbreviations.

At univariate analysis in HFrEF, EOV (214 patients) was discovered more frequently in those with advanced age and NYHA class, with more compromised systolic and diastolic function. Finally, in EOV was associated to worst exercise capacity (Table 2 ), i.e. in those with more reduced mean peak $\mathrm{VO}_{2}$, and steeper $\mathrm{VE} / \mathrm{VCO}_{2}$ slope (EOV was also found in $4 \%$ of CVD patients with LVEF dysfunction in class NYHA I with preserved mean peak VO2: mean peak $\mathrm{VO}_{2}$ was $=19 \pm 4,7 \mathrm{ml} / \mathrm{kg} / \mathrm{min}$.)

At multivariable logistic analysis for EOV in HFrEF patients. Five resting clinical variables were selected: age, LVEF, DecT, NYHA class, body mass index, and cardiac rhythm at the moment of CPET (Table 3). EOV was more commonly seen in older patients, in advanced NYHA class, in those with more depressed LVEF, and shorter DecT, and in sinus rhythm.

\section{Discussion}

Main findings were: 1) Kremser's EOV was noticed only in CVD and HFrEF patients; 2) of note, EOV was observed only in patients with impaired left ventricular (LV) systolic function; 3) percentage of EOV was superior in HFrEF patients; 4) rarely, EOV occurs in HFrEF patients with preserved exercise capacity (peak $\mathrm{VO} 2>14 \mathrm{ml} / \mathrm{kg} / \mathrm{min}$.); 5) at multivariate analysis in HFrEF, demographic and clinical variables were selected, and those with older age, advanced NYHA class, more depression of systolic and diastolic function in the presence sinus rhythm at time of CPET were more prone to develop EOV.

Only one experience calculated EOV prevalence, though in healthy subjects with a broad range of cardiovascular risk factors: in the EURO-EX trial [4]., EOV was detected in 17\%, and it was associated with a poor CPET performance and altered gas exchange profile. Although not proven, EOV can "unmask" a higher level of risk, not readily apparent when assessing aerobic capacity [4]. In our experience, a mixture of healthy subject and CVD patients was enrolled, with healthy subject less represented. In contrast with EURO-EX trial, EOV definition and CPET interruption criteria were dissimilar. Thus, these two experiences are poorly comparable; nevertheless, EOV was detected only in CVD patients with LV dysfunction and/or in HFrEF. Thus, we confirm that EOV might be related to hemodynamic derangement or ventilatory instability during exercise [12-15].

EOV has been shown to be a strong predictor of mortality in $\mathrm{HFrEF}$ [1-3]. In this setting, prevalence is widespread, ranging from 7 to $51 \%$ [16-28]. The variance of EOV might originate from the heterogeneity of its definition and different techniques of measures [29]: four original EOV descriptions [10,17,26,29] have been rec- ommended but many modifications are allowed. These four original EOV accounts have been reported in 23, 13, 6 and 2 experiences, respectively, with a mean prevalence of $28 \%, 37 \%, 35 \%$ and $50 \%$.

A "relatively" low prevalence of EOV was documented in HFrEF, in our study. Generally, we used to prescribe CPET when clinical stable condition, early mobilization or exercise training, and HF-saving therapeutic goals have been attained [30-32]. On the contrary, CPET is postponed in more disabled patients, i.e. in those with overt HFrEF or those with concomitant diseases, with advanced disease, disable and with complex clinical status or altered acid-base/ionic disequilibrium [12-14,33]. Thus, CPET is usually recommended only for the 'best' ones.

EOV was also noticed in those with preserved exercise capacity, with mean peak $\mathrm{VO}_{2}$ of more than $14 \mathrm{ml} / \mathrm{kg} / \mathrm{min}$.: these shocking findings solicitate further investigations. Finally, EOV appears more frequently in elderly HFrEF patients [33].

\section{Limitations}

Limitations deserve mention: i) EOV was defined, according to Kremser et al.'s criteria [10]; other EOV definition's criteria might produce different rate of occurrence; ii) prevalence recorded from a single center data-base could be limited, as CPET protocol modality, execution type and criteria of for exercise testing termination were homogeneous.

\section{Conclusions}

Kremser's EOV was observed in patients, and in those with CVD and systolic function impairment. Moreover, as EOV impacts prognosis in HFrEF patients, its occurrence can modify prognostic-decision models; in HFrEF, it is important to identify those are more prone EOV. However, more studies are needed to tackle the EOV prevalence in different CVD conditions and in normal subjects.

\section{References}

1. Corrà U. Exercise oscillatory ventilation in heart failure. Int $\mathbf{J}$ Cardiol 2016;206;S13-5.

2. Cornelis J, Taeymans J, Hens W, et al. Prognostic respiratory parameters in heart failure patients with and without exercise 
oscillatory ventilation-a systematic review and descriptive meta-analysis. Int J Cardiol 2015;182:476-86.

3. Corrà U, Piepoli MF, Adamopoulos S, et al. Cardiopulmonary exercise testing in systolic heart failure in 2014: the evolving prognostic role: a position paper from the committee on exercise physiology and training of the heart failure association of the ESC. Eur J Heart Fail 2014;16:929-41.

4. Guazzi M, Arena R, Pellegrino M, et al. Prevalence and characterization of exercise oscillatory ventilation in apparently healthy individuals at variable risk for cardiovascular disease: A sub-analysis of the EURO-EX trial. Eur J Prev Cardiol 2016;23:328-34.

5. Yajima T, Koike A, Sugimoto K, et al. Mechanism of periodic breathing in patients with cardiovascular disease. Chest 1994;106:142-6.

6. Ponikowski P, Voors AA, Anker SD, et al. 2016 ESC Guidelines for the diagnosis and treatment of acute and chronic heart failure. Eur J Heart Fail 2016;18:891-975.

7. Mezzani A, Corrà U, Bosimini E, et al. Contribution of peak respiratory exchange ratio to peak VO2 prognostic reliability in patients with chronic heart failure and severely reduced exercise capacity. Am Heart J 2003;145:1102-7.

8. Tabet JY, Beauvais F, Thabut G, et al. A critical appraisal of the prognostic value of the VE/VCO2 slope in chronic heart failure. Eur J Cardiovasc Prev Rehabil 2003;10:267-72.

9. Wasserman K, Jansen JE, Sue DY, et al. Principles of exercise testing and interpretation, $3^{\text {rd }}$ Editedion. Lippincott Williams \& Wilkins; 1999, p. 143-165.

10. Kremser CB, O'Toole MF, Leff AR. Oscillatory hyperventilation in severe congestive heart failure secondary to idiopathic dilated cardiomyopathy or to ischemic cardiomyopathy. Am J Cardiol 1987;59:900-5.

11. Giannuzzi P, Imparato A, Temporelli PL, et al. Dopplerderived mitral deceleration time of early filling as a strong predictor of pulmonary capillary wedge pressure in postinfarction patients with left ventricular systolic dysfunction. J Am Coll Cardiol 1994;23:1630-7.

12. Piepoli MF, Ponikowski PP, Volterrani M, et al. Aetiology and pathophysiological implications of oscillatory ventilation at rest and during exercise in CHF. Do Cheyne and Stokes have an important message for modern-day patients with heart failure? Eur Heart J 1999;20:946-53

13. Murphy RM, Shah RV, Malhotra R, et al. Exercise oscillatory ventilation in systolic heart failure: an indicator of impaired hemodynamic response to exercise. Circulation 2011;124: 1442-51.

14. Khoo MCK. Determinants of ventilatory instability and variability. Resp Physiol 2000;122:167-82.

15. Asmussen E. Regulation of respiration: 'the black box'. Acta Physiol Scand 1977;99:85-90.

16. Corrà U, Giordano $\mathrm{A}$, Bosimini $\mathrm{E}$, et al. Oscillatory ventilation during exercise in patients with chronic heart failure. Clinical correlates and prognostic implications. Chest 2002;121:1572-80.

17. Leite JJ, Mansur AJ, de Freitas HF, et al. Periodic breathing during incremental exercise predicts mortality in patients with chronic heart failure evaluated for cardiac transplantation. J Am Coll Cardiol 2003;41:2175-81.

18. Corrà U, Pistono M, Mezzani A, et al. Sleep and exertional periodic breathing in chronic heart failure: prognostic importance and interdependence. Circulation 2006;113:44-50

19. Guazzi M, Arena R, Ascione A, et al. Exercise oscillatory breathing and increased ventilation to carbon dioxide production slope in heart failure: An unfavorable combination with high prognostic value. Am Heart J 2007;153:859-67.

20. Guazzi M, Raimondo R, Vicenzi M, et al. Exercise oscillatory ventilation may predict sudden cardiac death in heart failure patients, J Am Coll Cardiol 2007;50:299-308.

21. Arena R, Myejrs J, Abella J, et al. Prognostic value of timing and duration characteristics of exercise oscillatory ventilation in patients with heart failure. J Heart Lung Transplant 2008;27:341-7.

22. Ingle L, Isted A, Witte KK, et al. Impact of different diagnostic criteria on the prevalence and prognostic significance of exertional oscillatory ventilation in patients with chronic heart failure. Eur J Cardiov Prev Rehab 2009;16:451-6.

23. Corrà U, Mezzani A, Giordano A, et al. Exercise haemodynamic variables rather than ventilatory efficiency indexes contribute to risk assessment inchronic heart failure patients treated with carvedilol. Eur Heart J 2009;30:3000-6.

24. Guazzi M, Boracchi P, Arena R, et al. Development of a cardiopulmonary exercise prognostic score for optimizing risk stratification in heart failure: the $(\mathrm{P}) \mathrm{e}(\mathrm{R}) \mathrm{i}(\mathrm{O})$ dic $(\mathrm{B})$ reathing during (E)xercise (PROBE) study. J Card Fail 2010;16:799-805.

25. Corrà U, Mezzani A, Giordano A, et al. A new cardiopulmonary exercise testing prognosticating algorithm for heart failure patients treated with beta-blockers. Eur J Cardiov Prev Rehab 2011;16:1-7.

26. Sun XG, Hansen JE, Beshai JF, Wasserman K. Oscillatory breathing and exercise gas exchange abnormalities prognosticate early mortality and morbidity in heart failure. J Am Coll Cardiol 2010;55:1814-23.

27. Corrà U, Giordano A, Mezzani A, et al. Cardiopulmonary exercise testing and prognosis in heart failure due to systolic left ventricular dysfunction: a validation study of the European Society of Cardiology Guidelines and Recommendations (2008) and further developments. Eur J Cardiov Prev Rehab 2011;19:32-40.

28. Arena R, Guazzi M, Myers J, et al. Exercise oscillatory ventilation reflects diminished quality of life and perceived functional capacity in patients with heart failure. Int $\mathrm{J}$ Cardiol 2011;153:213-4.

29. Cornelis J, Beckers P, Vanroy C, et al. An overview of the applied definitions and diagnostic methods to assess exercise oscillatory ventilation: a systematic review. Int $\mathrm{J}$ Cardiol 2015; 190:161-9.

30. Zurek M, Corrà U, Piepoli MF, et al. Exercise training reverses exertional oscillatory ventilation in heart failure patients. Eur Respir J 2012;40:1238-44.

31. Castro RR, Antunes-Correa LM, Ueno LM, et al. Reversal of periodic breathing after aerobic training in heart failure. Eur Respir J 2010;35:1409-11.

32. Apostolo A, Giusti G, Gargiulo P, et al. Lungs in heart failure. Pulmonary Medicine 2012;2012:952741.

33. Faisal A, Webba KA, Guenettec JA, et al. Effect of age-related ventilatory inefficiency on respiratory sensation during exercise. Respir Physiol Neurobiol 2015;205:129-39. 